\title{
Medications of Elderly in a Pharmacists' Perspective
}

\author{
Sambhu Ramesh"1, Manas Joshi Mallikasheril2 ${ }^{2}$ Stephy Paravaragath Justin ${ }^{1}$ \\ 1Department of Clinical Pharmacy, Acharya \& B M Reddy College of Pharmacy, Bengaluru, Karnataka state, INDIA. \\ ${ }^{2}$ Clinical Pharmacist, Aster Medicity, Kuttisahib Road, Near Kothad Bridge, South Chittoor, Cheranalloor, Kochi, Kerala 682027, INDIA.
}

\begin{abstract}
Context: Elderly population is one of the less studied areas in health sciences. Management of elderly health issues needs special consideration in all aspects right from the problem identification. India's elderly population is growing very fast, it is expected that elderly population in India will increase by $270 \%$ by 2050 . Hence health related problems of elderly in India needs special attention. Objective: To analyze the status of geriatric population in India by focusing on health status on the basis of demography, incidence of diseases, use availability and appropriateness of medications and role of pharmacist in reducing the inappropriateness in elderly care. Materials and Methods: Systematic review of 50 articles conducted. Participants with less than 60 years, articles from other countries discussing geriatric issues other than medications were excluded. Results and Discussion: There exists diversity in disease and health conditions on the basis of demography. Diversity also exists in medicine use among inpatients and outpatients. Major medication related problem was due to inappropriateness in medication use. Pharmacist intervention in geriatric improves the total care and rationality of medications. Conclusion: Better health status results were in urban elders. Implementation of national health programs to support elderly health care can extend good quality geriatric care to rural areas as well. Extending clinical pharmacist services can significantly improve rational use of medicines in geriatric population.

Key words: Geriatric population, Demography, Pharmacist's intervention, Medication related problems.
\end{abstract}

\section{INTRODUCTION}

Ages nearing or surpassing the life expectancy can be called as Elderly or old age. It is the last phase of any individual's life. Any person in this phase of ageing had a progressive deterioration of all body functions, which can lead to diverse physical and psychological problems making their daily life activities troublesome. WHO report on ageing states that, proportion of world's population over the age of 60 will be expected to double from $11 \%$ to $22 \%$. This proportion will be greatly affected by growing economies like India, China and Brazil. The report also points that majority of mortalities from developing countries were due to non-communicable diseases. This denotes the extent of sufferings of elderly from chronic non-communicable diseases will be much higher in developing economies compared to developed counterparts. ${ }^{1}$ It is found that
$6 \%$ of older people in the developing world are victims of some sorts of maltreatment including denial of medical services. The major difference of treatment of elderly people in developed and developing countries is, when developed countries need to examine the safety of existing medical setups, the developing counterpart need availability of medication setups for the need of elderly. Safety and availability of medication in elderly thus gains importance. ${ }^{2}$

Around the world, geriatric population is increasing. It is accompanied by decline in the proportion of young. It is estimated that by the year of 2050 , the number of older person in the world will exceed the number of young for the first time in history. The health of older persons typically deteriorates with increasing age, including greater demand for long term care as the numbers of oldest old grow. ${ }^{3}$
DOI: 10.5530/ijopp.9.4.8

Address for correspondence: Dr Sambhu Ramesh Department of Pharmacy Practice, Acharya \& B M Reddy College of Pharmacy, Bengaluru, Karnataka-560107. Phone: +91-8861104425. Email: ramesh.sambhu@ gmail.com 
India, the world's second largest population is undergoing a transition in its demography. There is a notable decrease in the fertility rate from 3.5 in 1991 to 2.6 in 2011. Average life expectancy rises from 58.6 in 1991 to 65 in 2011. This shows our success in dealing with childhood diseases, maternal mortality and helping women to achieve control over their fertility. However this transformation disturbs our health economy by lacking a good quality geriatric service especially at primary level.

Elderly population faces many problems, which include problems related to health, social support and economic insecurity. Their healthcare needs differs from young counterparts. Medication related problems in elderly are one of the most studied preventable health related problems in elderly. Polypharmacy and irrational use of medicines are common problems in geriatric prescription. Incidence and severity of adverse drug reactions are two to three times higher in elderly and most of them are potentially avoidable. ${ }^{4,5}$

\section{METHODS}

A systematic review of published literature was performed upto august 2015 using standard databases NCBN, Google scholar, PubMed. Search strategies included concepts like Elderly population, medication related problems in elderly, medication use pattern in Geriatrics, role of Pharmacist in improving geriatric care and health related quality of life of elderly. Articles available as open access and using all study methods were included, articles from other countries describing. Articles in language other than English, participants with age below 60 and articles from countries other than India focusing a special geriatric condition apart from medications were excluded. The interventions suggested in articles included formulation of geriatric specific guidelines, including geriatrics into medical curriculum and introducing pharmacist into geriatric clinical team. Additional studies were identified through reference and citation tracking. Only full text articles were taken for inclusion process. Literature search for geriatrics yielded a total of 50 papers. After applying the exclusion criteria 5 articles mentioning demography of geriatrics in all over India, 11 articles mentioning demography and health status in specific state. 3 articles mentioning medication related problems in elderly. Three articles mentioning suggestions for improving the practice of medication in elderly. Three articles mentioning role of pharmacist for improving geriatric medical practice and one article mentioning pharmacoeconomic considerations in elderly were included in this review. No specific methodological quality assessments were performed.

\section{RESULTS}

Results showed that well distinguished variation in health related issues exist among geriatrics of rural and urban geriatric population. Apart from health related problems, geriatric population also suffers serious social, financial and spiritual issues. ${ }^{5}$

\section{Demographic studies}

A Report from central statistics and program implementation says that $65 \%$ of aged has to depend on others for their daily needs and the condition is even worse with elderly females, only $14-17 \%$ is economically independent. Report also identifies that prevalence of heart disease among elderly men and women was higher in urban areas than in rural. Urinary problems were more commonly seen among males and joint related problems reported more with females. Multiple disabilities are more reported from rural areas. Locomotors disability reported in $3 \%$ of the rural elderly. Report also identifies that apart from physical illness, aged are more likely to be victims of poor mental health which arises from senility, neurosis and extent of life satisfaction. The study also mentions about the state wise variation seen in various demographic parameters. The study points out that one fourth of the total elderly are free from all diseases whereas one out of two elderly in India suffers from at least one chronic disease that needs long term care. ${ }^{1}$

Another similar study points out that the unconditional respect, power and authority that the elderly people used to enjoy in external traditional families is being gradually eroded in India in recent years. Various trends explain that the changes in the living arrangements including decline in fertility leaving fewer children available to take care of the older parents and rural to urban migration that separates families. The problems identified from the study were lack of coverage of various insurance schemes and non-availability of insurance schemes for those who aged above 65 . The study also focus that majority of the geriatric care services available in the country were in urban areas and a majority of elderly who needs special care are living in rural areas. The intervention suggested was specialized geriatric care centers should be opened in the rural areas of the country. ${ }^{3}$ 
An Indian study discussing social issues among elderly people of India points that more than $20 \%$ of Indian population smoke daily, twice as many people living in rural area smoke everyday compared with urban counterpart. This explains the predisposing factor for respiratory diseases in rural area compared with urban.,

\section{Studies focusing role of Pharmacist}

An American systematic review discussing the drug therapy in elderly points that one quarter of the hospital admissions for people aged 65 years and older are associated with medication related problems typically ADR's or non-compliance. Factors contributing to these factors in elderly are Polypharmacy, unsupervised use of non-prescription medicine, prescribing of medications not suitable for adults and inadequate counselling and education of patients about their medications. Study reports that multiple disease states are one of the major factors contributing to poly medicine. Factors contributing to non-adherence are taking several medicines with different schedules, cost of medication, delays in ordering medication refills and inability to travel to pharmacy. ${ }^{7}$ One major suggestion came from this study was government regulations for medication safeguards including mandates for drug regimen review donot exists for older individuals outside of extended care setting. Intervention suggested from this study is a well-functioning pharmacist with in depth knowledge in geriatrics at these extended care facilities can promote safer prescribing and enhance medical compliance. An Interventional Study identifies that majority of drug related problems identified were prescriber related (55\%) and drug related (39.6\%). Study also identifies that digoxin and NSAID use were associated with highest risk of potential drug related problems. Study recommends that a well-functioning clinical pharmacist can play vital role in reducing drug related problems in elderly. ${ }^{8,9}$

\section{State wise analysis of health status of elderly}

A cross-sectional study from India points that majority of elderly are from socioeconomically lower class and were unable to purchase their medications. The study finds that most prevalent health problems among elderly were depression, osteoarthritis, hypertension, and cataract. Study mentions that all health related problems seen among the population were treatable. ${ }^{10}$ Another cross-sectional study from south India points that assessing the correct age was difficult as many donot remember their exact date of birth neither were their records available. Study finds that most commonly addressed problem was anemia $(52.5 \%)$ and nearly $90 \%$ of the elderly were having no regular source of income. Study concludes that illness in elderly are multiple and chronic in nature. Another study from North India on nutritional data reveals $26 \%$ of male and $46.5 \%$ females are under weight. ${ }^{11}$ Study from Kerala shows that older people have high predisposition to psychiatric diseases and much of them go undiagnosed. A systemic review confirms that there exists a wide variation in the disease states from state to state and elderly are more prone to abuse and avoidance. Study points that elderly in India suffer from dual medical problems, communicable and non-communicable diseases along with impairment of special sensory functions. ${ }^{12}$

\section{Use of medication and related problems}

Proportion of higher use of medicines were more common in $75-84$ years, and among them $33.2 \%$ were taking more than 4 drugs and $13 \%$ were taking 6 or more drugs. A study on institutionalized patients says that several drugs can be avoided in elderly without any significant negative effects on mortality, morbidity and quality of life and with benefits in financial consequences. Study also points that much of the evidence based drugs are inappropriate with greater risks and lower benefits with institutionalized patients. A systematic review from Finland states that medication use among elderly is a potential cause for falls. The study finds that psychotropic medicines and other CNS drugs are responsible for increased fall in elderly, the study recommends that more RCT's are needed and also falls should be considered as an important adverse reaction and should be included in various guidelines intended for geriatrics. Study on drug utilization evaluation states that antiplatelet drugs (93\%) are the most commonly used drug among geriatrics followed by $\mathrm{H}_{2}$ blockers $(77.22 \%)$, anti-emetics $(67.6 \%)$, vasodilators $(55 \%)$ and hyperlipidemia drugs (52\%). Study also identifies that ADR is most commonly seen in patients on anti-diabetic and anti-bacterial therapy. Another prescription evaluation study states that aspirin is the most commonly prescribed drug in adults and atenolol is the most frequently used drug in elderly. Study on potentially inappropriate medication finds that $23.58 \%$ received at least one drug which was potentially inappropriate and $7.422 \%$ drugs prescribed inappropriately. Yet another study on prescribing pattern of elderly identifies that antimicrobial are the most commonly prescribed drugs (16.94\%). Respiratory system drugs were most com- 
mon (38\%) followed by cardiovascular (15\%), Gastro intestinal (13\%) and infectious diseases (12\%). A study to find rationality of drugs for elderly says that $30 \%$ of hospital admissions in elderly are due to drug related problems and also finds that $52 \%$ of prescriptions were inappropriate. Study found that $39.7 \%$ of prescriptions had one interaction, $44.2 \%$ of prescriptions had 2 interactions and $14.7 \%$ had 3 interactions and $1.47 \%$ had four or more interactions. Study identifies that poly pharmacy is seen in $20-40 \%$ of older people. Study recommends that rational drug therapy is essential in reducing potentially inappropriate medication use. A study to assess nature and frequency of drug related problems in elderly inpatients found that medication related problems are identified in $56 \%$ of the patients studied and among them cardio vascular medications accounts for $37 \%$ of medication related problems followed by nervous system drugs (19\%) and insulin (9.4\%)..$^{13-19}$

\section{DISCUSSION}

Various studies found that health status among elderly people was significantly lower compared to any other age groups. There exist variations in incidence of disease, availability of good quality care and mode of care received on the basis of sex, dependence status and demography. All geriatric studies from different states shows need for an indigenous specialized care services. Communicable diseases were reported commonly in rural population with a higher predisposition to males. Smoking and other habits play a major role for this. Hence controlling social habits among rural population is important. Immobility and sedentary life style among urban adult results in an increased cardio vascular problems in males and joint related problems in females. This shows importance of physical activity and exercises. Even though many fitness centers like gym, meditation, yoga centers exists in urban areas none of them gives a special attention to elderly population.

Another major factor to be considered is disability, locomotor disability, loss of hearing and blindness were commonly seen among elderly. Physiological changes due to ageing results in reduced bone mineral density, loss of muscle mass and reduced control of body parts. This results in a significant risk of increased fall and an increased risk of fractures among elderly. Progressive loss of vision and reduced hearing capacity will further affect the cognitive ability of elderly. This scenario brings the importance of assessing the disability status of elderly. ${ }^{20}$ Disability status assessment should be done in elderly with a special focus to rural adults, since much of the rural adults go unregistered even to government programs. Assessing the correct age was difficult for many adults in rural areas. This is due to lack of records regarding their birth, non-enrollment in government programs etc... this situation is more common in rural females. Socio economic status of the family is another important consideration. Measuring socio economic status brings the social picture of the family. Modified B.G. Prasad Classification can be used to assess the SES among elderly in rural areas. Socio economic studies found that most of the rural adults fall into the category of class III and class IV. This showed that financial conditions of elderly were poor and they completely depends on others for their daily needs. Unfortunately this scale does not measures any other constrains like occupation education status etc., Mistreatment, chronic verbal abuse, financial abuse, physical abuse and neglect were increasingly seen among elderly. Rural elderly females suffered most compared to others. This highlights the need for social empowerment of rural elderly women and spreading awareness among the working population. . $^{1,219}$

Incidence of various diseases among elderly varies from state to state, however this scenario is more seen among the rural elderly compared to their urban counterparts. Incidence and prevalence of many of the diseases among elderly are not well studied. There exists great variation from state to state, some states needs to address from basic issues like providing proper nutrition, proper diagnosing of medical condition and availability of basic medicines. Undiagnosed psychiatric conditions are another major issue with geriatrics. Psychiatric disorders were one of the commonly diagnosed disorders among elderly in western countries. Depression and anxiety were the most commonly diagnosed. However in India psychiatric disorders among elderly were less studied and less diagnosed..$^{12}$ Much of the psychiatric disorders among elderly were classified as age associated problems. Prescribing for psychiatric disorders is another major hurdle, many of the psychiatric medications are classified as potentially inappropriate for elderly by many guidelines.

Medication use among elderly varies significantly on the basis of demography. Drugs for metabolic disorders like anti hyperglycemic, cardio vascular drugs were commonly prescribed in urban males. Drugs for hormone related problems and joint disorders were prescribed commonly in urban females. It is found that even though there is no considerable variation in the incidence of chronic diseases between 
rural and urban elders, underutilization of medication is seen in rural adults. Drugs for respiratory diseases were more commonly seen in rural elders. Antimicrobials were the most commonly prescribed drugs for elderly at hospitals. It is found that many elders are on chronic treatment with NSAIDs. There were no studies available to evaluate the medication adherence, medication related problems and proper medication use and prescription refilling. ${ }^{21-23}$

Foreign studies comparing the role of pharmacists prove that intervention from a pharmacist can improve drug therapy of elderly very significantly. Many countries have formulated guidelines to promote safe medication use among elderly. Beer's criteria for identifying the potentially inappropriate medication among elderly was the first intervention made to improve drug therapy in elderly. Studies' comparing the effectiveness of various criteria's found that STOPP and START criteria's shown to have better efficiency in identifying and preventing potentially inappropriate medication among elderly. One study from India identified that intervention by the pharmacist can improve drug therapy in elderly patients. This study highlights the ability of pharmacists in improving drug therapy, it also adds that a clinical pharmacist in Indian health setup can significantly reduce the potential drug related problems in elderly. ${ }^{24,25}$

\section{CONCLUSSION}

After critical evaluation of available resources, it is found that better quality of life, good socio economic standards and better utilization of available health care resources were seen in urban elders. Significant variation is seen in the type of disease and use of medication among elders on the basis of sex and demography. Unidentified problems, unmet medical needs, lack of availability of neassary medications were the serious problems addressed by rural elders. Drugs for metabolic disorders are used mostly by urban elders whereas respiratory medicines and drugs for communicable diseases were seen mostly in rural elders. Major challenges for caring elderly include demographic diversity in the incidence of diseases, inappropriateness and errors in prescriptions and lack of national health programs to uplift elderly care. Currently no geriatric guidelines are practiced in India. There is only one study from India statistically proves interventions from clinical pharmacist can improve medication use in elderly. Many studies from other countries support this context. Hence, a clinical pharmacist can intervene and reduce medication related problem in elderly. There is a need for much more research in the field of medication use in special population like elderly in India.

\section{ACKNOWLEDGEMENT}

We thank Mr. Binai Kochunni Sankar for his support and guidance.

\section{CONFLICT OF INTEREST}

No conflict of interest are declared.

\section{ABBREVIATIONS USED}

WHO: World health organization; NCBI: National center for biotechnology information; ADR: Adverse drug reaction; NSAID: Non-steroidal antiinflammatory drug; CNS: Central nervous system; RCT: Randomised control trial; SES: Socio-economic status; STOPP: Screening tool for older people prescriptions; START: Screening tool to alert to right treatment.

\section{REFERENCES}

1. Central statistics office, Ministry of statistics and program implementation Government of India. June 2011. Situation analysis of elderly in India.

2. S. Irundaya R. July 2006. Population ageing and health in India. Center for enquiring into health and allied themes.

3. Abhimanyu M, Anushree R. Indian elder: factors affecting geriatric care in India. Global Journal of Medicine and Public Health. 2013;2:1-5.

4. Population reference bureau, March 2012. Today's research on aging. Program and policy implementation.

5. Population division, Department of economic and social affairs United Nations. 2002. World population ageing:1950-2050.

6. Vikram P, Somnath C, Dan C, et al. chronic diseases and injuries in India. The Lancet. 2011;377:413-28.

7. Sole-Auro A, Pierre-Carl M, Michael D. Hurd, Eileen Crimmins. Disease Incidence and Mortality Among Older Americans and Europeans Rand population and ageing. 2013 [working paper].

8. California state board of pharmacy, communication and public education committee. 2003. Drug therapy considerations in older adults.

9. Identification of potential drug related problems in Elderly; the role of community pharmacist.

10. Frequency and nature of drug related problems in elderly

11. Anil J, Joy B, Malini K. et al. Morbidity pattern among the elderly population in the rural area of Tamil Nadu, India. Turk J Med Sci. 2006;36(1):45-50.

12. Samson SRN, Sai TSR. Estimation of life style diseases in elderly from a rural community of Guntur district of Andra Pradesh. Journal of Clinical and Diagnostic Research. 2014;8(4):JC01-JC04

13. Muhammed E, Irundaya RS, Kumar KA, et al. Gender and mental health in kerala. Institute of Social Studies Trust and Center for Developmental Studies.

14. Sirpa H, Eija L, Kirsti L. Medication as a risk factor for falls: critical systematic review. Journal of Gerontology. 2007;62A(10):1172-81.

15. What is Polypharmacy? National prescribing service newsletter[internet] December 2000. [cited September 2015]. Available from.

16. Doron G, Zur-gil S, Joshna BI. The war aginesh Polypharmacy: A new cost effective geriatric palliative approach for improving drug therapy in disables elderly people. Israyel Medical Association Journal. 2007;9(6):430-34.

17. Jenifer MS, Josiden CW, Thomas GB, et al. Predicting adverse drug reactions in older adults; a systematic review of the risk prediction models. Clinical Interventions in Ageing. 2014;9:1581-93. 
18. Sushma T, Sinha AK, Patwardhan K, et al. Prevalance of health problems among elderly : A study in a rural population of Varanasi. Indian J Prev Soc Med. 2010;41(3):226-30.

19. Gopal KI. Anita Nath Geriatric health in India: concerns and solutions. Indian J Community Med. 2008;33(4):214-18.

20. Medical and socio-economic problems faced by elderly.Strategies to improve the QOL of the elderly; the role of the health care system. Role of pharmacist in the provision of palliative care. The pharmacy guide of Australia. Department of health and ageing. Australian Government.

21. Binit NJ, Tejas KP, Manish JB, et al. Drug utilization pattern and pharmaco economic analysis in geriatric medical In Patients of a tertiary care hospital of India. Journal of Pharmacology and pharmacotheraputics. 2014;5(1):15-20.
22. Neha S, UmaA, Shobha K, et al. Screening of prescriptions in geriatric population in a teaching hospital in North India. The Journal of Phytopharmacology. 2013;2(5):38-45.

23. Zaveri HG, Mansuri SM, Patel VJ. Use of potentially inappropriate medicines in elderly: A prospective study in medicine out patient department of a territory care teaching hospital. Indian J Pharmacology. 2010;42(2):5-98.

24. Bhogan C, Shanmugham S, Anandavalli SN, et al. Rational use of drugs in elderly at a private corporate hospital. Universal Journal of Pharmacology. 2013;42(2):95-8.

25. Veena DR, Padma L, Sapna P. Drug prescribing pattern in elderly patients in a teaching hospital. Journal of Dental and Medical Sciences. 2012;1(5):39-42. 